\title{
Construction Technology of Prestressed Cable Truss System of Gymnasium in YueQing
}

\author{
Mingmin Ding ${ }^{1, a^{*}}$, Bin Luo ${ }^{2, b}$, Yuzhou Shen ${ }^{3, c}$ and Yangjie Ruan ${ }^{4, d}$ \\ ${ }^{1}$ Department of civil engineering, Southeast University, Nanjing 210096, China \\ ${ }^{2}$ National pre-stressed engineering center of China, Nanjing 210096, China \\ ${ }^{3}$ Key Laboratory of Concrete and Pre-stressed Concrete Structures of the Ministry of Education, \\ Nanjing 210096, China \\ aemail: andyming1989seu@foxmail.com, bemail: seurobin@seu.edu.cn, \\ cemail: 36489452@qq.com, 'email: 15298373086@163.com
}

Keywords: cable truss; prestress; cable; simulation calculation

Abstract: A cable truss support system was used in the roof of the gymnasium of YueQing sports center. It is composed by the grid structure of the monolayer surface and the cable truss support system. The prestress construction is difficult by its deformation and stress control. Based on the cable truss system of gymnasium in YueQing, construction technologies for relaxing cable, hanging cable and tension equipment are introduced. Accurate simulating calculation of the process of prestress construction is done by ANSYS. In this article, authors analyzed nonlinear features of this structure in the construction process .Cable force, deformation and steel structure stress were simulating analyzed in order to ensure the process of construction.

\section{Introduction}

Cable truss support system is a new type of prestressed tension structure developed in recent years. Analysis of large-span prestressed structure construction contents includes force-finding analysis, zero state-finding analysis, temperature impact analysis, construction process analysis, assembling analysis and construction control analysis. Construction methods include installation method and tensioning method. Tensioning equipment includes jack and tooling, etc. In this paper, whole process of tracking simulation analysis of prestressed construction is made by a nonlinear finite element software ANSYS, using YueQing Gymnasium as background. This paper identifies a reasonable tensioning construction program, guides the actual construction process and ensures the smooth progress of the project.

\section{Structural features}

The prestressed steel stadium used in this article is a model of prestressed cable truss system. It is made of single-layer reticulated structure and cable truss system. Its roof support system includes $\mathrm{V}$-shaped columns and ring beams.

Cable Truss system must add prestress to obtain a greater structural rigidity to bear loads and maintain the shape. Therefore, prestress force is also an important part of structure, besides geometric parameters, geometric topological relations and connecting nodes. 'Force' and 'Shape' of this kind of structure are unified, 'Force' is a balance on the corresponding 'Shape'. So, it's necessary to control both 'Force' and 'Shape' during the prestress construction.

The cable truss system of gymnasium in Yueqing includes external radial cable (ER-x in short), internal radial cable (IR-x in short), external ring cable, internal ring cable, constructed cable and slings. All of them are made of flexible cables, connect with each other through both ends of cables and the corresponding node pins. 


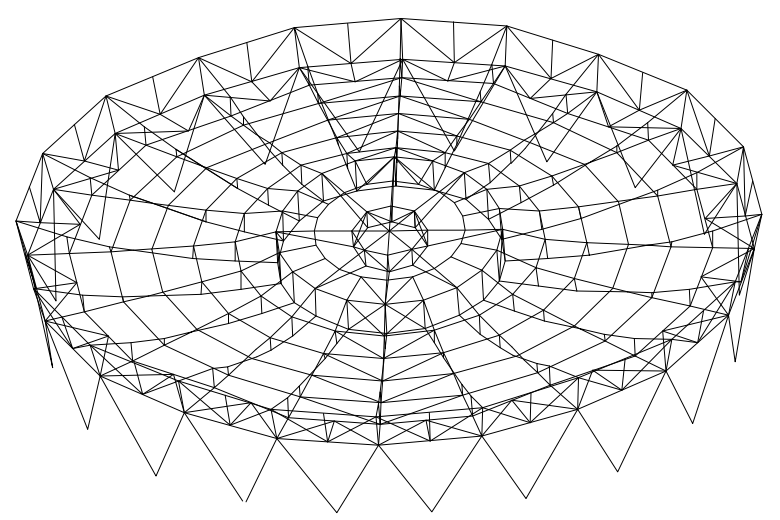

Fig. 1 Isometric view of main stadium

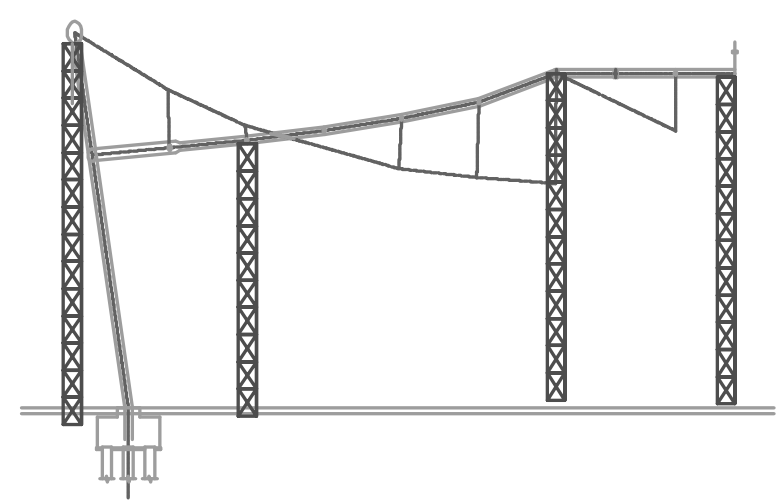

Fig. 2 Schematic diagram of V-shaped columns and radial steel beam support bracket node

\section{Construction programs}

Overall program. The prestressing project is divided into four stages, designed to add the cable force to $10 \%, 50 \%, 90 \%$ and $100 \%$, Where in each stage is divided into eight batches. Tension sequence shown in Table 1.

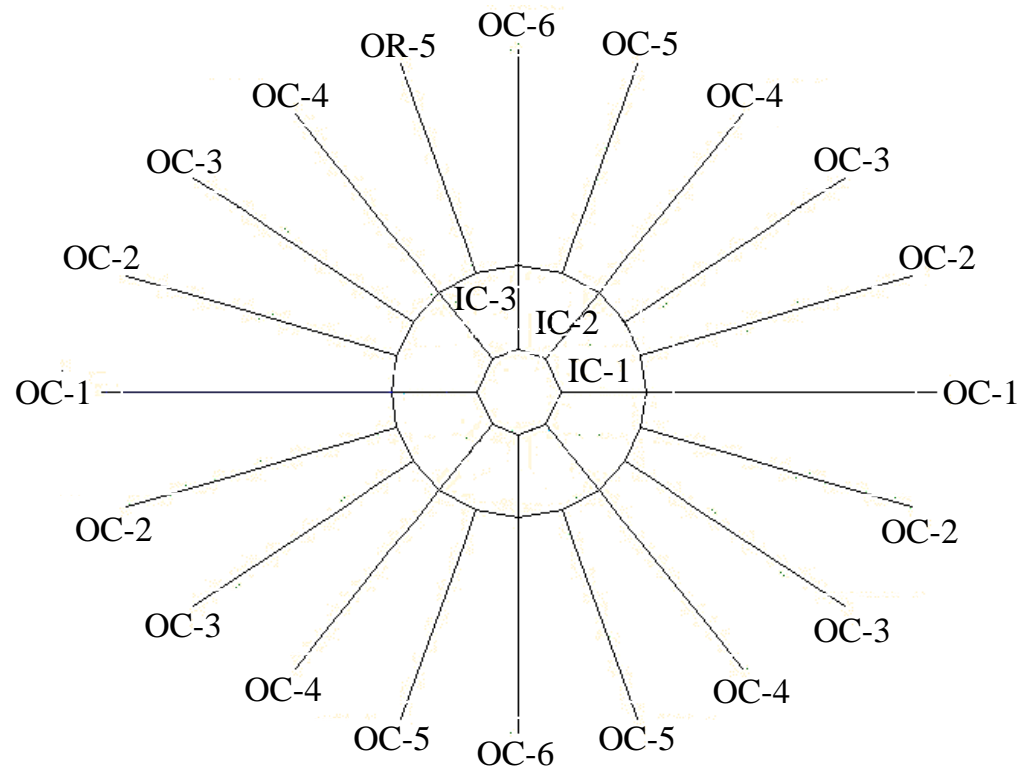

Fig. 3 Schematic diagram of Cable Ids

( IC denotes inner olique cables, and OC denotes outer oblique cables)

Table 1 Tension sequence of each stage

\begin{tabular}{ccccccccc}
\hline Tension batch & Batch 1 & Batch 2 & Batch 3 & Batch 4 & Batch 5 & Batch 6 & Batch 7 & Batch 8 \\
\hline \multirow{2}{*}{ Cable IDs } & OC-1 & OC-4 & OC-5 & OC-2 & OC-6 & OC-3 & IC-1 & IC-2 \\
\hline
\end{tabular}


Overall project construction sequence shows below.

(1) The supporting tire rack is set up on the middle of roof to prepare for assembling single-layer reticulated structure.

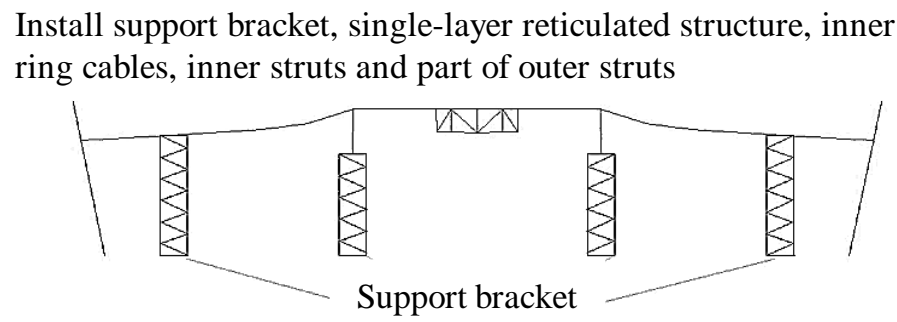

(2) Installing inner ring cables, inner poles, inner radial cables, outer radial cables and part of outer poles in turn.

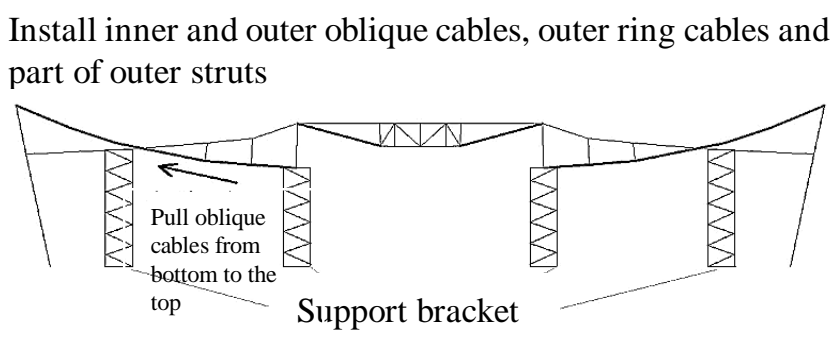

(3) Installing the rest of all.

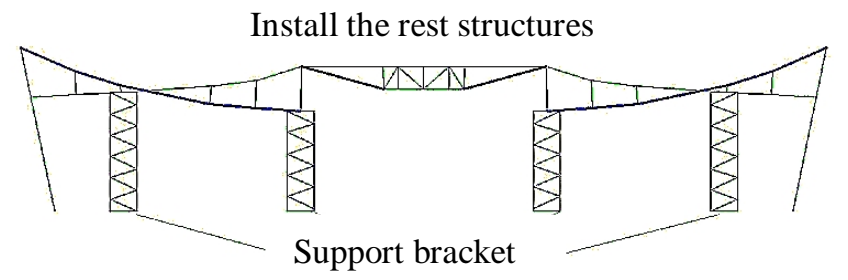

(4) According to the structure characteristics and the results of calculation and analysis, we decide to tension radial cables to get prestress.

Tension outer oblique cables in batches

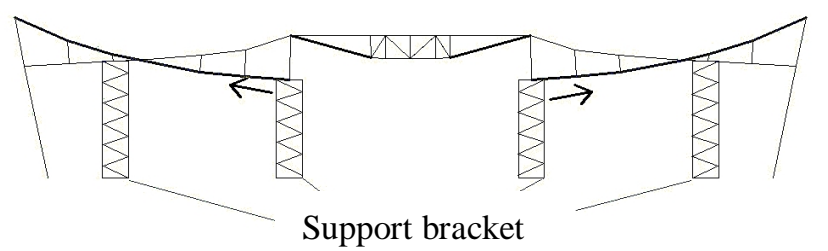

Tension inner oblique cables in batches

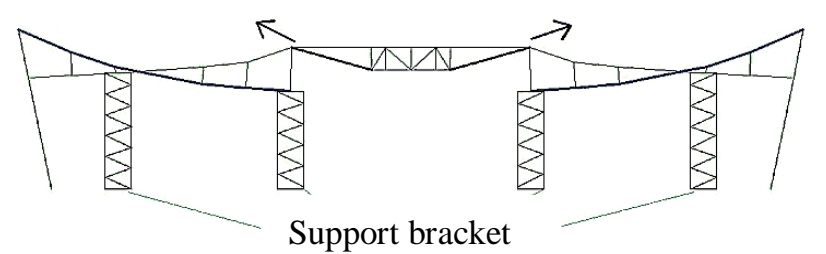

(5) After tensioning cables, it's time to installing Roof panels and so on.

\section{Prestressed construction program.}

(1)Install cables

1) Installing slings, radial cables and ring cables from the inner to the outer in turn.

2) In the same ring, installing slings first, then cable clips and radial cables, ring cables at last.

3) Before installing cables, measuring the actual distance of cables to consider structural deviations and adjust the cable length.

4) Cable installation sequence

Determining and producing cables with prestress under the condition of initial prestress. Marking cable clamp position on the surface of radial cables. $\rightarrow$ Transporting cables to the site. $\rightarrow$ Installing slings and upper clips. $\rightarrow$ Measuring the distiance between the sling node (both upper ones and lower ones) and the radial sling nodes, and ensure the installing deviations $\rightarrow$ Adjusting the installing lengths of radial cables and installing radial cables (ensure the yaw directions and yaw sizes of slings being the same in the same ring) $\rightarrow$ Installing ring cables according to the markers on the surfaces (don't tighten the cable clips so as to allow relative sliding between ring cables and cable clips) $\rightarrow$ Completing ring cables pretension (ensure the cable force and dip of every cable segment in the same ring being uniform)。 
(2) Tension cables

1) Tension inner and outer radial cables in several stages and several batches. Tension outer cables in the first stage and tension inner cables in the second one.

2) Classify the same batch cables and tension them at the same time

To control the overall shape of the structure, to ensure the uniformity of tensioning, the same batch cables are classified into five parts and tensioned at the same time, of which the first four parts are controlled by tension stroke when tensioning, the last one is controlled by cable force.

3)Tension points

The cable collets connecting inner radial cables and the third ring ring-beams. The cable collets connecting outer radial cables and upper ring-beams.

4) Controlling project and target of tensioning

The controlling includes two aspects, the controlling of cable force and the controlling of displacement. Wherein, the controlling of cable force is more important.

\section{Whole construction process simulation}

Before tensioning, the structures of prestressed cable truss system do not get good overall stiffness. Thus, uncontrolled change of structure displacement and steel stress will happen without detailed analysis of structure characteristics. So, it's necessary to do whole process construction simulation analysis of prestress, analyze the structure response of construction process and guarantee the security of construction.

The establishment of finite element model. Using ANSYS to build the finite element model. The steel beams and exterior steel columns use Beam188 unit, the struts use Link8 unit, the cables use Link10 unit (only for tension), the supporting tire rack use Link10 unit (only for compression). Elasticity modulus of main structure is $2.06 \times 10^{5} \mathrm{MPa}$, poisson ratio is 0.3 , thermal expansion coefficient is $1.2 \times 10^{-5}$. Elasticity modulus of cables is $1.9 \times 10^{5} \mathrm{MPa}$, thermal expansion coefficient is $1.2 \times 10^{-5}$. Construction load consider the weight of structure.

Construction process simulation. The analysis considers large deformation and stress stiffening effect taking into accout the characteristics of the nonlinear structure (geometic and material nonlinearity). This project uses Equivalent Temperature Difference Method to simulate prestress. The structure is applied sequentially equivalent temperature to simulate prestressed tension according to the formulated batch and tension sequence.

Table 2 Cable equivalent temperature

\begin{tabular}{ccccccccccc}
\hline Cable position & OC-1 & OC -2 & OC -3 & OC -4 & OC -5 & OC -6 & OC -7 & IC-1 & IC -2 & IC -3 \\
\hline $\begin{array}{c}\text { Target cable } \\
\text { force }[\mathrm{kN}]\end{array}$ & 1826 & 1800 & 1747 & 1692 & 1631 & 1609 & 1643 & 336 & 389 & 336 \\
$\begin{array}{c}\text { Equivalent } \\
\text { temperature }\left[{ }^{\circ} \mathrm{C}\right]\end{array}$ & -123 & -121 & -115 & -108 & -103 & -102 & -104 & -58 & -61 & -58 \\
\hline
\end{tabular}

Construction process analysis. Tracking the structure response of tension process, including cable force, displacement and steel stress, etc.

Table 3 The simulation results

\begin{tabular}{ccccc}
\hline Construction Phase & $10 \%$ & $50 \%$ & $90 \%$ & $100 \%$ \\
\hline OC-1 force [kN] & 209.86 & 1140 & 1690 & 734.7 \\
Mid-span vertical displacement [mm] & -23.08 & 137.3 & 409.8 & 472.6 \\
Steel maximum stress [Mpa] & 38.50 & 111.7 & 178.7 & 207.3 \\
\hline
\end{tabular}




\section{Conclusion}

The cable truss support system is a new kind of hybrid space structure system. Based on the summarization of previous research results, Jiangsu Culture Sports Center gymnasium as the engineering background to the actual structure construction, construction technology of similar ellipse suspen-dome is mainly studied. Finite element analysis, stability performance, whole construction process analysis, optimization design of prestressed and Optimum analysis of steel nodes are systematically studied.

The author draws some conclusions of the cable truss support system structural performance, and the problems that should be studied further are put forward.

\section{Acknowledgement}

Project supported by the Twelfth Five Year National Science And Technology Support Program under Grant from the China Academy of Building Research (No. 2012BAJ03B06), the National Natural Science Foundation of China (No. 51308105), A Project Funded by the Priority Academic Program Development of Jiangsu Higher Education Institutions (PAPD), and the Fundamental Research Funds for the Southeast University (No. KYLX_0152, SJLX_0084, KYLX_0149)

\section{References:}

[1] Bin Luo, Zhengxing Guo, Research and Application of Large-span Space Steel Structures Prestress Construction Technology — Prestress Construction Technology Analysis of Large-span Steel Space Structure, J. Construction Technology. 341 (2010) 101-106.

[2] Zhengxing Guo, Bin Luo. Research and Application of Large-span Space Steel Structures Prestress Construction Technology — Packaged Technology of Prestress Construction for Large-span Steel Space Structures, J. Construction Technology. 344 (2011) 96-102.

[3] Zhengxing Guo, Jun Qiu, Bin Luo, The Construction Technology of Elliptical Spokewise Prestressed Cable-truss Structure, J. Construction Technology. 7(2005) 95-101.

[4] Li Mei, Xu Ruilong, Qin Jie, Li Guoli, Qian Yingxin, Zhao Qingli, Prestress Construction Technology of Beam String Spatial Structure at Beijing Financial Street Seasons Place, J. Construction Technology. 37 (2008) 141-143.

[5] Chen Guan-dong, Huang Cheng-yong, Ruan Miao, Construction Technology of Prestressed Steel Beam String Structure of Culture and Sports Center in Sanmenxia. 12 (2011) 1075-1077.

[6] Valdek Kulbach, Half-span loading of cable structures, J. Journal of Contrutional Steel Research. 49 (1999) 167-180.

[7] Valdek Kulbach, Half-span loading of cable structures, J. Journal of Contrutional Steel Research. 49 (1999) 167-180. 